\title{
The Recurrent Flush: A Case and Review of Systemic Mastocytosis
}

Wasim Mansoor MD, Shaan Chugh MD, FRCPC, Carlo V. Hojilla MD PhD FRCPC, Rashmi S. Goswami MD PhD FRCPC FCAP, Rodrigo B. Cavalcanti MD MSc FRCPC

\section{About the Authors}

Wasim Mansoor, Shaan Chugh, and Rodrigo B. Cavalcanti are with the Department of Medicine, Division of General Internal Medicine, University of Toronto. Carlo Hojilla is with the Department of Pathology and Laboratory Medicine, Sinai Health System and the Department of Laboratory Medicine and Molecular Diagnostics, Sunnybrook Health Sciences Centre.Rashmi Goswami is with the Department of Hematology and Transfusion Medicine, University Health Network and the Department of Laboratory Medicine and Pathobiology, University of Toronto.

Corresponding Author: wasimmansoor@gmail.com

Submitted: August 10, 2017; Accepted: January 22, 2018. Published: June 25, 2018.

\begin{abstract}
Systemic Mastocytosis (SM) is a hematologic neoplasm characterized by an abnormal proliferation of mast cells, which have the potential to infiltrate one or more visceral organs. Patients can present with a wide constellation of symptoms making it a challenging diagnosis for clinicians. Non-specific symptoms such as fatigue, headache, and weight loss may predominate; however, some patients may present with acute onset of urticaria, flushing, and diarrhea. Due to its rarity, clinicians often face a challenge in evaluating, diagnosing and effectively treating SM. Identification during the indolent phase is important as SM can progress to aggressive leukemias or myeloproliferative disorders. In this article, we present a case of SM, and discuss current practices in diagnosis, evaluation and management. We conclude with future directions for treatments and diagnosis.
\end{abstract}

\section{RESUME}

La mastocytose systémique (SM) est une néoplasie hématologique caractérisée par une prolifération anormale de mastocytes, qui ont le potentiel d'infiltrer un ou plusieurs organes viscéraux. Les patients peuvent présenter une vaste gamme de symptômes, ce qui en fait un diagnostic difficile pour les cliniciens. Les symptômes non spécifiques tels que la fatigue, les maux de tête et la perte de poids peuvent prédominer. Cependant, certains patients peuvent présenter un début aigu d'urticaire, de rougeur et de diarrhée. En raison de sa rareté, les cliniciens sont souvent confrontés à un défi dans l'évaluation, le diagnostic et le traitement efficace de la SM. Lidentification au cours de la phase indolente est importante car la SM peut évoluer vers des leucémies agressives ou des troubles myéloprolifératifs. Dans cet article, nous présentons un cas de SM, et discutons des pratiques actuelles en matière de diagnostic, dévaluation et de gestion. Nous concluons avec les orientations futures pour les traitements et le diagnostic.

\section{Case Presentation}

\section{Clinical History}

A 48-year-old woman presented with syncope and hypotension with a blood pressure of 50/27 $\mathrm{mm} \mathrm{Hg}$. The patient was not known to have any chronic medical illness and was on no routine medications at home. She was aggressively fluid resuscitated followed by development of pulmonary edema. She was intubated and transferred to the ICU where she required ongoing vasopressor therapy. Over the next few days, she was

DOI: 10.22374 /cjgim.v13i2.234 
hemodynamically stabilized and returned to her usual state of health. The patient was discharged after 3 days and asked to follow-up with an allergy and immunologist specialist; however, allergy testing was negative. Over the next few months, the patient continued to have episodic feelings of fatigue, chest heaviness, increased warmth, and associated dyspnea. She also described intermittent episodes during which she would develop an erythematous rash involving the face and chest (Figure 1) with associated facial flushing and headaches that would spontaneously resolve. These were found to be triggered by red wine, exercise, sunlight exposure, while having no discernible association with her menses.
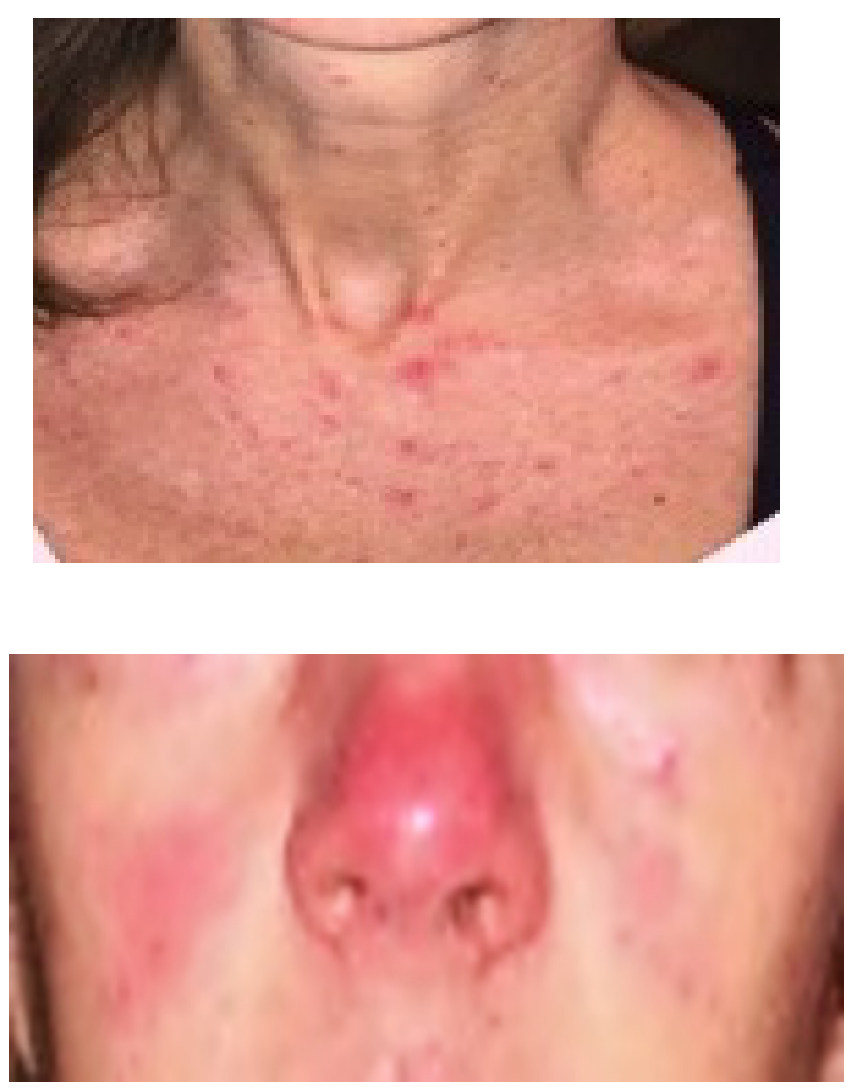

Figure 1. Image of patient showing an erythematous rash involving the face and chest with associated facial flushing and headaches that would spontaneously resolve.

\section{Laboratory and Investigations}

The patient was eventually referred to the internal medicine clinic at University of Toronto University Health Network where she was thoroughly investigated. The following investigations were performed:where she was thoroughly investigated. The following investigations were performed:
- Serum and urine metanephrines and 5-HIAA: negative.

- Serum tryptase levels (during acute exacerbation episodes): negative.

- Complete blood count: normal range.

- Echocardiogram and colonoscopy: negative for carcinoid tumour.

- Serum immunoglobulins: normal range.

- CT abdomen: no evidence of any underlying lymphadenopathy, organomegaly, or malignancy. 
Despite the absence of elevated serum tryptase levels, the patient's constellation of symptoms was concerning for an underlying diagnosis of SM. Recently published guidelines suggest serum KIT mutation analysis if there is a suspicion of SM despite normal tryptase levels (30); however, this was also found to be within normal range.

The patient was conservatively managed with daily Zantac and Claritin; however, episodes continued to get more frequent and debilitating, prompting a bone marrow biopsy to exclude SM. Biopsy and aspiration results revealed SM with presence of a KIT D816V mutation.

Histopathological reports showed that the bone marrow showed 20\% hypocellularity, and immunohistochemical staining for tryptase was positive in mast cells (Table 1; Figure 4).

\section{Management and Therapy}

A total skeletal survey was performed to rule out bony lesions. She was started on vitamin D and calcium supplements for maintaining bone health. Due to the continued recurrence of her symptoms she was started on ketotifen and Aspirin which resulted in symptomatic improvement. Hematology consultation suggested no systemic therapy was needed, given the relatively low levels of mast cells demonstrated on histopathology.

\section{Patient Experience}

"New Years Eve 2014 was my first major incident. I ended up in the ER in anaphylactic shock after a home cooked meal and some wine. Subsequent allergy testing was negative so the event was deemed idiopathic anaphylaxis and I was told to carry Epi-pens. For the following three months I kept having allergic type reactions, often after a full day of activities and dinner. The reactions consisted of major facial flushing, nasal congestion, hives across chest, increased heart rate with sometimes chest pains/palpitations., resulting in frequent trips to the ER. After a significant episode, typically I would have the shakes and a severe headache followed by days or weeks of extreme exhaustion, diarrhea, and brain fog. I spent many days in bed just too tired to get up, with a very heavy feeling in my body like my muscles were lead weights. In hindsight now I also recall breaking out in red spots across my abdomen and thighs after spending time in a hot tub or hot bath. My family doctor referred me to several different allergists/immunologists and an endocrinologist. Many tests were run but all were normal. By April 2015 I started to feel better so we decided it was an unexplained event that cleared on its own. Then in December 2015, I started

Table 1. Histopathological Report for the Patient

\section{Histology:}

Histological evaluation of the specimen provided shows a hypocellular (20\%) bone marrow with readily apparent mast cells on the aspirate, which are mostly rounded in morphology with a few spindled and many (>25\%) degranulated forms present. Lymphoid infiltrates are readily identified on the squash preparation and the trephine biopsy, associated with linear groups of spindled mast cells in a perivascular distribution.

Immunohistochemical Staining:

Tryptase: Positive in mast cells; subset show spindling and are found in a linear distribution (>15 mast cells)

CD2, CD25 and CD117: Positive in atypical mast cells

Flow Cytometry

Bone marrow aspirate demonstrates a normal proportion of CD34+ blasts, comprised of myeloid blasts and hematogones, showing normal maturation patterns. In addition, there is an aberrant population of CD117+ mast cells that are positive for CD2 and CD25. 


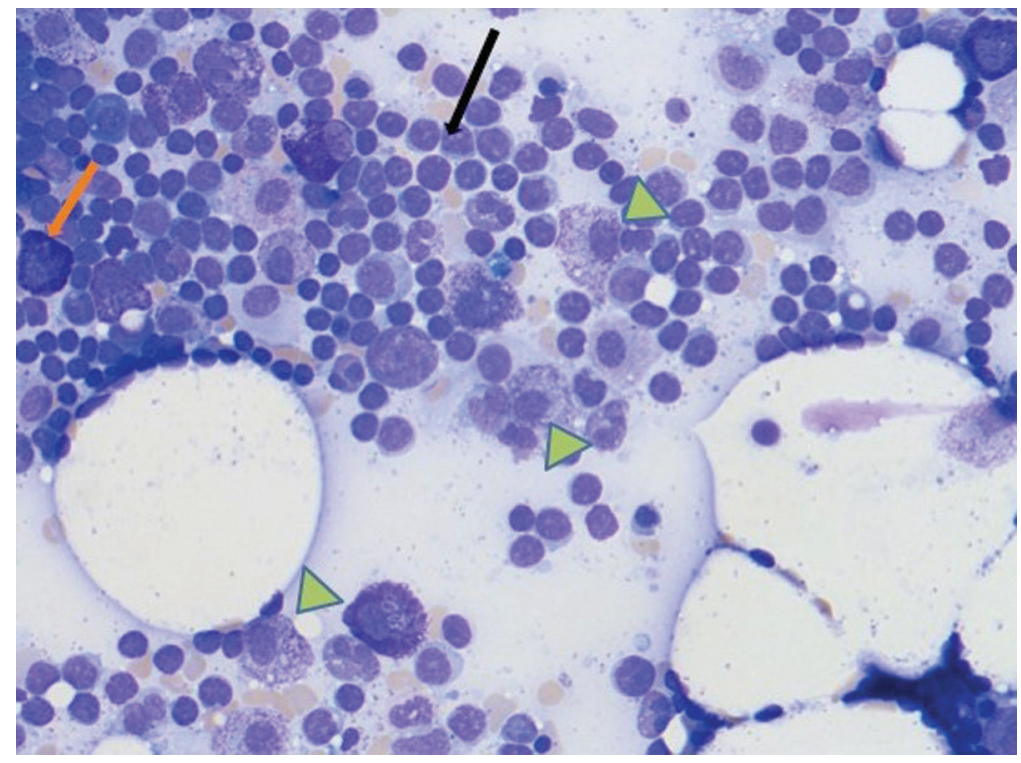

Figure 4. Bone marrow aspirate: Normal mast cells with deep purple granulation and round contours are indicated by the orange arrow. Green arrows indicate atypical mast cells with round contours but reduced granulation. Background lymphocytes (small round, blue cells) are indicated by the black arrow. This is a squash preparation May-Grunwald-Geimsa stain, 600x magnification.

having the flares again and after minor surgery in January 2016, I had a severe reaction in the recovery room, quite similar to the New Year's Eve experience. This provided a new piece to the puzzle since I was sedated and hadn't eaten anything yet had an anaphylactic type reaction. Armed with this new information and a referral to new team of Internal Medicine doctors, I was given a bone marrow biopsy in February 2016 and a conclusive diagnosis of SM.

All mastocytosis patients seem to react to different triggers. Currently my biggest triggers are heat, alcohol, high histamine foods (i.e., tomatoes, chocolate, aged/fermented foods etc.), emotional and physical stress (including talking intensely and being overtired) and exercise. In other words, I feel like I am allergic to my life and all of the things that I enjoy. I have had to completely change my life in order to try and control these triggers in addition to taking a daily regime of antihistamines and mast cell stabilizing drugs. I have had to significantly modify my work, food, exercise, talking, travel, medical/dental procedures, and social activities. However, through careful trigger management and medication, I am often able to control the symptoms and have a good day with moderate activity levels. But this is nowhere near the activity level of my life prior to mastocytosis. If I am feeling good and return to my desired activity level, then I get a flare up of symptoms. Though the medications seem to suppress the extent of the reaction, I have been able to manage these flares by resting and taking the time for my body to recover. It can take days or weeks until I feel well again. Managing the mastocytosis is a daily task for me and mentally exhausting. I lack confidence to do many things that I used to as I am afraid of an anaphylactic reaction. I just never know how or if I will react to things, so I approach everything with caution and mindfulness.

While having a diagnosis has been helpful in many regards, it is a difficult diagnosis as it is a lonely disease to have since many doctors do not have direct experience in this area. The daily management is challenging and largely trial and error on my part. Definitive answers to questions I have been difficult to come by as every patient with mastocytosis has different triggers and different levels of reactions. I am hopeful that as we continue to get a better handle of how to manage this disease, I will find new ways to enjoy my new life as a patient living with SM."

\section{DISCUSSION}

\section{Clinical Manifestation}

$\mathrm{SM}$ is a rare disease that can go unrecognized due to its protean nature coupled with its rarity, making it a challenging diagnosis for even the most astute clinician. As the name suggests, this disease is characterized by the pathological accumulation of mast cells in one or more tissues. Historically the disease is classified in 2 major forms: cutaneous mastocytosis (CM), and $\mathrm{SM}$. CM is a disease that primarily affects children and is a benign self-limiting disease which often resolves by puberty. In contrast, SM mostly afflicts adults and symptoms tend to be more persistent. ${ }^{1}$ Symptoms can range from non-specific symptoms 
such as weight loss, diarrhea, and fatigue, to specific symptoms consisting of pancytopenia, osteoporosis, and gastrointestinal symptoms. In severe cases, a second hematological process can concomitantly occur resulting in a secondary myeloproliferative disorder, myelodysplastic syndrome, myelofibrosis or acute leukemia. Table 2 contains a comprehensive list of symptoms patients with SM may report.

Table 2. Tissue Involvement and Clinical Symptoms Associated with SM

\begin{tabular}{|l|l|}
\hline Skin & pruritis, flushing, urticaria pigmentosa \\
\hline Gastrointestinal & $\begin{array}{l}\text { abdominal pain, diarrhea, duodenal ulcers (PUD), } \\
\text { protein-losing enteropathy }\end{array}$ \\
\hline Cardiovascular & $\begin{array}{l}\text { palpitations, syncope, pre-syncope, anaphylaxis } \\
\text { (hypotension, angioedema) }\end{array}$ \\
\hline Neurological & $\begin{array}{l}\text { depression, anxiety, cognitive impairment, } \\
\text { peripheral neuropathy }\end{array}$ \\
\hline Musculoskeletal & $\begin{array}{l}\text { osteopenia, secondary osteoporosis, fractures, } \\
\text { chronic bone pain }\end{array}$ \\
\hline Constitutional & night sweats, fatigue, anorexia, weight loss, fever \\
\hline Organ Infiltration & $\begin{array}{l}\text { bone marrow (pancytopenia), lymphadenoapthy, } \\
\text { hepatosplenomegaly }\end{array}$ \\
\hline
\end{tabular}

As noted in Table 2, symptoms associated with SM are broad and non-specific. Skin manifestations are the hallmark of CM but are also a common presentation seen in SM, especially in indolent systemic mastocytosis (ISM). Urticaria pigmentosa is one of the many skin manifestations which presents itself as brown papules and are indicative of systemic disease in $10-70 \%$ of cases of SM. ${ }^{2-4}$ Mast cell (MC) infiltration in the dermis can also lead to flushing, pruritis, and urticaria, thought to be secondary to spontaneous release of MC mediators. Interestingly, some patients may be able to reproduce cutaneous manifestations when experiencing surface friction, a phenomenon known as Darier Sign. ${ }^{5}$

Anorexia is also common and is thought to be caused by persistent diarrhea and enteropathy and can often be mistaken for irritable bowel syndrome. Additionally, due histamine's role in acid production, patients are also at risk for developing duodenal ulcers and may need to be prophylactically treated with a proton pump inhibitor. It is estimated that GI bleeding occurs in $11 \%$ of SM. Contrary to previously reports, it is estimated that peptic ulcer disease is under-diagnosed. In a recent prospective study, four of nine patients with SM had peptic ulcer disease on upper GI endoscopy. ${ }^{6}$ Neurological manifestations include depression, anxiety, and cognitive impairment and are often underrecognized. The proposed mechanism of these manifestations include MC degranulation leading to central nervous system (CNS) neuroinflammation ${ }^{7}$ Constitutional symptoms are rare in ISM, unless the disease has progressed to advanced SM (ASM), or mast cell leukemia (MCL). Extracutaneous MC infiltration is the hallmark of SM when compared to CM. Commonly affected organs include the bone marrow, liver, lymph nodes, and spleen. Due to bone marrow involvement, hematological abnormalities are common. Anemia is present in $50 \%$ of patients with SM, and is more prevalent in aggressive forms of SM when compared to ISM. Eosinophilia is seen in only roughly $15 \%$ of patients. ${ }^{7,8}$

Increased MC degranulation can also have effects on the MSK system. Patients with SM can experience widespread body aches and myalgias which can often be misdiagnosed as fibromyalgia. A study reported that roughly $31 \%$ of SM patients may experience similar symptoms. ${ }^{8}$ Furthermore, it was noted in a cohort study that $50 \%$ of patients with SM had evidence of bone involvement on imaging, with osteoporosis being the most common finding. ${ }^{10}$ The risk of developing osteoporosis-related fractures, although higher in aggressive forms of SM, were also found in $37 \%$ of patients with ISM. ${ }^{11}$

\section{Diagnosis and Classification}

As with many orphan diseases, the incidence and prevalence of SM is unknown due to a lack of adequate epidemiological studies. The only population-based study was a retrospective cohort conducted in Denmark that examined 548 patients with SM between 1997-2010. Incidence of SM was estimated to be 0.89 per 100,000 people, with a cumulative incidence of 12.46 for every 100,000 people, and a 14 -year prevalence of 9.59 per $100,000 .^{2}$ The most common subtype identified in the study was indolent systemic mastocytosis (ISM) including urticaria pigmentosa, followed by SM with subtype unknown. ${ }^{2}$

As understanding of this has disease evolved, the World Health Organization released consensus diagnostic criteria and a classification system for the diagnosis of SM and revised them in 2016 (Tables 3 and 4). ${ }^{12,13}$

Once a diagnosis of SM is made, the disease is classified based on severity to determine prognosis and the appropriate treatment. According to the revised 2016 WHO classification there are 5 major subtypes of SM: Indolent SM (ISM), Smoldering SM (SSM), SM with associated hematologic neoplasm (SMAHN), Aggressive SM (ASM), and MC Leukemia (MCL). The classification is based on bone marrow findings coupled with the presence or absence of B and C Findings (see Table 4). ${ }^{12}$ 
The Recurrent Flush

Table 3. Diagnostic Criteria

\section{A. Criteria $^{(a)}$}

1. Major Criteria: Multifocal, dense infiltrates of mast cells (>15 MCs in aggregates) detected in sections of BM and/or other extracutaneous organs

\section{Minor Crtieria:}

a. In biopsy sections of BM or other extracutaneous organs, $>25 \%$ of MCs in infiltrate are spindle shaped or have atypical morphology or, of all MCs in BM aspirate smears, $>25 \%$ are immature or atypical.

b. Detection of activating point mutation at codon 816 of KIT in BM, blood or other extracutaneous organ.

c. MCs in BM, blood or other extracutaneous organs express CD2 and/or CD25 in addition to normal MC markers (CD117).

d. Serum total tryptase persistently exceeds $20 \mathrm{ng} / \mathrm{mL}$ (unless there is associated clonal myeloid disorder, in which case this parameter is not valid).

Abbreviations: $\mathrm{ANC}=$ Absolute neutrophil count, $\mathrm{BM}=$ Bone marrow, $\mathrm{MC}=$ mast cell

aDiagnosis: 1 major +1 minor or 3 minor

Table 4. WHO Classification of Systemic Mastocytosis

\section{B. Findings}

a. BM biopsy shows $>30 \%$ infiltration by MCs (focal, dense aggregates) and/or serum total tryptase level $>200 \mathrm{ng} / \mathrm{mL}$.

b. Signs of dysplasia or myeloproliferation in non-MC lineage, but insufficient criteria for definite diagnosis of hematopoietic neoplasm (AHN) with normal or slightly abnormal blood counts.

c. Hepatomegaly without impairment of liver function, and/or palpable splenomegaly without hypersplenism, and/or lymphadenopathy on palpation or imaging.

\section{Findings}

a. BM dysfunction manifested by 1 or more cytopenias (ANC $<1.0 \times 10^{9} / \mathrm{L}$, hemoglobin $<10 \mathrm{~g} / \mathrm{dL}$ or platelets $<100 \times 10^{9 / \mathrm{L}}$ ) but no obvious non-MC hematopoietic malignancy.

b. Palpable hepatomegaly with impairment of liver function, ascites and/or portal hypertension.

c. Palpable splenomegaly with hypersplenism.

d. Malabsorption with weight loss due to gastrointestinal MC infiltrates.

\section{Indolent Systemic Mastocytosis (ISM)}

Meets criteria for systemic mastocytosis (SM). No C findings.

Smoldering Systemic Mastocytosis (SSM)

Meets criteria for SM, but with 2 or more B findings and no $C$ findings.

SM With Associated Clonal Hematologic Neoplasm (SM-AHN)

Meets criteria for SM and criteria for AHN as distinct entity per WHO classification.

Aggressive Systemic Mastocytosis (ASM)

Meets criteria for SM. One or more C-findings. No evidence of MC leukemia.

\section{Mast Cell Leukemia (MCL)}

Meets criteria for SM. Bone marrow biopsy shows a diffuse infiltration, usually compact, by atypical, immature MCs. Bone marrow aspirate smears show $>20 \%$ or more MCs. In typical MCL, MCs account for $>10 \%$ or more of peripheral blood white cells.

\section{Rare Variant}

Aleukemic mast cell leukemia, as above, but $<10 \%$ of white blood cells are MCs.

ANC = absolute neutrophil count; $\mathrm{BM}=$ bone marrow; $\mathrm{MC}=$ mast cell; $\mathrm{AHNMD}=$ associated hematological neoplasm

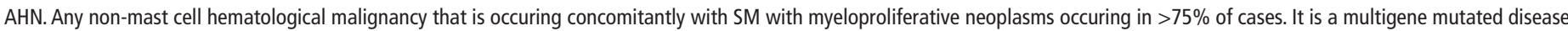
in which SM occurs as a late event. ${ }^{14}$ 
It is evident that diagnosis and classification of SM is challenging, and as such the above-mentioned diagnostic and classification algorithm was created to aid clinicians to properly diagnose and prognosticate patients with SM. However, some clinicians have proposed removing MCL and SM-AHN and placing them under myeloproliferative disorder due to similarities in morphology and prognosis. This proposal, however, has not been formally adopted as of yet. ${ }^{14}$

\section{Evaluation}

When suspecting $\mathrm{SM}$, it is necessary to take a thorough history and physical exam. Patients should be assessed for B-findings such as hepatomegaly, splenomegaly, lymphadenopathy or $\mathrm{C}$-findings such as ascites, cachexia, and pallor. Laboratory examination includes complete blood count looking for anemia, thrombocytopenia or pancytopenia. In more aggressive forms of the disease, liver enzymes may be elevated. If there is suspicion for bone involvement, serum calcium levels may be elevated. Appropriate imaging should be obtained based on clinical symptoms (i.e., X-rays and bone densitometry to rule out pathological fracture or osteoporosis and an abdominal CT to evaluate for organomegaly).

Identification of potential triggers is important as some patients can present with persistent, or episodic symptoms. Additionally, a serum tryptase level should be obtained to evaluate for MC degranulation. Elevations above $20 \mathrm{ng} / \mathrm{mL}$ in suspected patients clinches a diagnosis of SM. Therefore, tryptase levels can be used a screening tool in diagnosing more aggressive forms of SM. However, many patients with ISM have normal tryptase levels and thus serum tryptase screening may provide false negative results.

Although not part of the WHO diagnostic criteria, other surrogate markers of MC degranuation can also be useful in evaluating patients for SM. These include 24 hour urine N-methylhistamine $(\mathrm{NMH})$ and $11 \beta$-prostaglandin F2 $\alpha$ (BPG), which has shown to have a sensitivity of $71 \%$ and $53 \%$ respectively. ${ }^{15}$ Additionally, testing peripheral blood for presence of KIT mutations should be performed (minor criteria) as it correlates to disease severity. ${ }^{16,17}$ However, due to low sensitivity a negative result does not exclude disease if clinical suspicion is high.

Once appropriate data has been gathered and if there is a high pretest likelihood for disease, then the next step is bone marrow biopsy and aspirate. Biopsy and aspirate should be sent for total MC\% (stain for the receptor tyrosine kinase c-Kit (also known as CD117) and/or tryptase), CD25 and CD2 expression, and KIT mutation analysis to fullfill the diagnostic criteria for SM. The aspirate should also be examined by flow cytometry for CD30 and CD117. In patients with leukocytosis and/or eosinophilia, it is necessary to examine the bone marrow cells for the JAK2 V617F mutation as well as for $B C R / A B L 1$ and FIPL1/PDGRA rearrangements. ${ }^{1}$

Once a diagnosis of SM is made, patients should be classified in one of the subcategories based on the presence or absence of $\mathrm{B}$ and $\mathrm{C}$ findings (see Table 4). Treatment is then tailored based on the subtype of SM. Refer to Table 5 for diagnostic algorithm for suspected SM patients.

\section{Treatment and Prognosis}

Treatment of SM is based on limited clinical data due to paucity of evidence in the literature. We aim to briefly discuss standard therapies for various subtypes of SM, however the discussion regarding treatment will focus on treatments to he considered by a general internist.

\section{Indolent Systemic Mastocytosis}

Patients with Indolent Systemic Mastocytosis (ISM) have a normal life expectancy, and thus their treatment is primarily aimed at symptom management. ${ }^{18}$ Although rare, progression can occur in 1 to $5 \%$ of individuals. In one study, it was noted that patients with elevated serum B2-microglobulin and KIT mutation in myeloid and lymphoid cells were independent predictors of progression. ${ }^{19}$ It is important for these patients to keep a diary to identify any potential triggers of MC degranulation. Common triggers include: hymenoptera stings, alcohol (red wine most commonly), and medications (NSAID and Aspirin) have been reported in the past. ${ }^{20}$ Additionally, patients should be counseled to carry an epinephrine pen in case of an acute emergency.

First-line therapy revolves around mediator-related symptomatic control. Antihistamines can be used to ease flushing and pruritis, and may also relieve GI symptoms. Patients refractory to antihistamines may also benefit from Aspirin to control flushing as seen in our patient. ${ }^{21}$ It is important to note however that NSAIDs and ASA may also trigger symptoms in certain patients and thus careful evaluation must be taken when administering these drugs. ${ }^{20}$ Cromolyn sodium has been assessed in limited randomized control trials of patients with SM which showed improvement in GI symptoms. ${ }^{22}$ Omalizumab, a humanized IgG antibody against IgE has been demonstrated in limited case studies to reduce frequency of anaphylaxis in SM. As such, omalizimab can be used in patients who present with daily symptoms and whose disease has been refractory to maximum doses of antihistamines. ${ }^{23}$

When above-mentioned symptomatic control therapy fails, cytoreductive therapy can be considered. Interferon (IFN-alpha) and cladribine have shown good responses. ${ }^{24}$

Patients suspected of SM should also receive a skeletal survey and bone densitometry and be started on appropriate vitamin $\mathrm{D}$ and calcium supplements if they are unable to meet their daily 

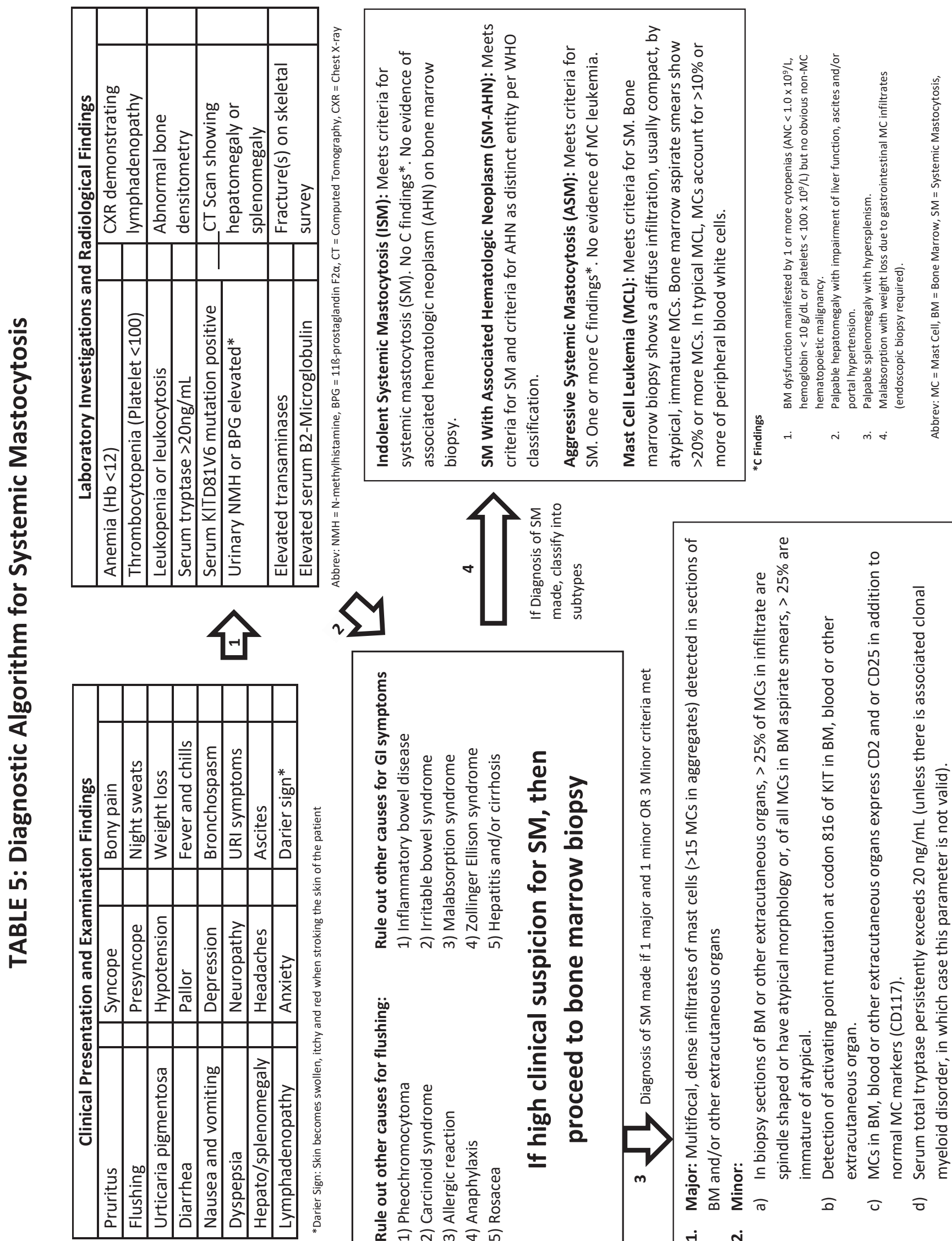
requirements through diet alone. Patients who are at high risk for osteoporosis should be evaluated on a case by case basis for consideration of bisphosphanates.

\section{Associated Hematologic Neoplasm, Aggressive SM, and MC Leukemia}

Patients with Associated Hematologic Neoplasm (AHN), Aggressive SM (ASM), and MC Leukemia (MCL) carry a poor prognosis compared to ISM due to their aggressive disease course. The median survival for aggressive SM is 41 months and for MCL it is less than 6 months. ${ }^{25}$ The prognosis for $\mathrm{AHN}$ is dependent on the concomitant hemotalogical malignancy present.

Hematopoetic stem cell transplantation (HSCT) is the most definitive treatment option that confers the potential for cure in appropriate patients. Based on a large retrospective study it was noted that patients with SM-AHN conferred a 74\% 3-year survival benefit with HSCT followed by 43 and 17\% for ASM and MCL patients, respectively. ${ }^{25-27}$ The strongest risk factor for worse overall survival was a diagnosis of MCL. As such, due to the paucity of treatment options for ASM and MCL, clinical trial drugs are often first line of therapy in patients. This continues to be an area of further research as the efficacy of new cytoreductive modalities are currently being investigated.

\section{Conclusion}

SM remains a rare disease with protean manifestations. Epigenetic studies are creating a better understanding of systemic mastocytosis, and tyrosine kinase inihibitors are currently being evaluated to address gain of function mutations of the c-Kit receptor in many variants of SM. The results of these studies are promising and could represent future avenues for treatment of ASM and MCL. More efforts need to be placed in creating standardized screening tools for early diagnosis. One promising approach is outlined in Table 5 . This could be followed by categorizing symptoms using a scoring system. This may help in establishing a pretest likelihood for the disease, to guide clinicians on pursuing bone marrow biopsy for early prognostication and treatment.

\section{REFERENCES}

1. Valent P, Sperr WR, Schwartz LB, et al. Diagnosis and classification of mast cell proliferative disorders: delineation from immunologic diseases and nonmast cell hematopoietic neoplasms. J Allergy Clin Immunol 2004;114:3-11.

2. Cohen SS, Skovbo S, Vestergaard H, et al. Epidemiology of systemic mastocytosis in Denmark. Br J Haematol 2014;166:521-8.

3. Soter NA. Mastocytosis and the skin. Hematol Oncol Clin North Am 2000;14:537-55, vi.

4. Moura DS. Neuropsychological features of adult mastocytosis. Immunol Allergy Clin North Am 2014;34:407-22.

5. Soter NA. Mastocytosis and the skin. Hematol Oncol Clin North Am 2000;14:537-55, vi.

6. Georgin-Lavialle S, Lhermitte L, Dubreuil P, et al. Mast cell leukemia. Blood 2013; 121:1285-95.
7. Lim KH, Tefferi A, Lasho TL, et al. Systemic mastocytosis in 342 consecutive adults: survival studies and prognostic factors. Blood 2009;113:5727-36.

8. Horny HP, Ruck M, Wehrmann M, et al. Blood findings in generalized mastocytosis: evidence of frequent simultaneous occurrence of myeloproliferative disorders. Br J Haematol 1990;76:186-93.

9. Lim KH, Tefferi A, Lasho TL, et al. Systemic mastocytosis in 342 consecutive adults: survival studies and prognostic factors. Blood 2009;113:5727-36.

10. Barete S, Assous N, de Gennes C, et al. Systemic mastocytosis and bone involvement in a cohort of 75 patients. Ann Rheum Dis 2010;69:1838-41.

11. Van Der Veer E, Van Der Goot W, De Monchy JGR, et al. High prevalence of fractures and osteoporosis in patients with indolent systemic mastocytosis. Allergy 2012;67:431-8.

12. Arber DA, Orazi A, Hasserjian R, et al. The 2016 revision to the World Health Organization (WHO) classification of myeloid neoplasms and acute leukemia. Blood 2016;127:2391-5.

13. Horny H, Metcalfe D, Bennet J, et al. Mastocytosis. In: Swerdlow S, Campo E, Harris N, et al, eds. WHO Classification of Tumours of Haematopoietic and Lymphoid Tissues. 4th ed. Lyon, France: World Health Organization; 2008.

14. Wang SA, Hutchinson L, Tang G, et al. Systemic mastocytosis with associated clonal hematological non-mast cell lineage disease: clinical significance and comparison of chromosomal abnormalities in SM and AHNMD components. Am J Hematol 2013;88:219-24.

15. Valent P, Arock M, Akin C, et al. The classification of systemic mastocytosis should include mast cell leukemia (MCL) and systemic mastocytosis with a clonal hematologic non-mast cell lineage disease (SM-AHNMD). Blood 2010;116:850-1.

16. Akin C, Metcalfe DD. Surrogate markers of disease in mastocytosis. Int Arch Allergy Immunol 2002;127:133-6.

17. Valent P, Horny HP, Escribano L, et al. Diagnostic criteria and classification of mastocytosis: a consensus proposal. Leuk Res 2001;25:603-25.

18. Valent P, Sotlar K, Sperr WR, et al. Refined diagnostic criteria and classification of mast cell leukemia (MCL) and myelomastocytic leukemia (MML): a consensus proposal. Ann Oncol 2014;25:1691-700.

19. Escribano L, Álvarez-Twose I, Sánchez-Muñoz L, et al. Prognosis in adult indolent systemic mastocytosis: a long-term study of the Spanish Network on Mastocytosis in a series of 145 patients. J Allergy Clin Immunol 2009;124:514-21.

20. Pardanani A, Lim KH, Lasho TL, et al. WHO subvariants of indolent mastocytosis: clinical details and prognostic evaluation in 159 consecutive adults. Blood 2010;115:150-1.

21. Brockow K, Jofer C, Behrendt H, et al. Anaphylaxis in patients with mastocytosis: a study on history, clinical features and risk factors in 120 patients. Allergy 2008;63:226-32.

22. Butterfield JH, Weiler CR. Prevention of mast cell activation disorder associated clinical sequelae of excessive prostaglandin D2 production. Int Arch Allergy Immunol 2008;147:338-43.

23. Horan RF, Sheffer AL, Austen KF. Cromolyn sodium in the management of systemic mastocytosis. J Allergy Clin Immunol 1990;85:852-5.

24. Carter MC, Robyn JA, Bressler PB, et al. Omalizumab for the treatment of unprovoked anaphylaxis in patients with systemic mastocytosis. J Allergy Clin Immunol 2007;119:1550-1.

25. Lim KH, Pardanani A, Butterfield JH, et al. Cytoreductive therapy in 108 adults with systemic mastocytosis: outcome analysis and response prediction during treatment with interferon-alpha, hydroxyurea, imatinib mesylate or 2-chlorodeoxyadenosine. Am J Hematol 2009;84:790-4.

26. Ustun C, Reiter A, Scott BL, et al. Hematopoietic stem-cell transplantation for advanced systemic mastocytosis. J Clin Oncol 2014; 32:3264-74.

27. Paul C, Sans B, Suarez F, et al. Masitinib for the treatment of systemic and cutaneous mastocytosis with handicap: a phase 2a study. Am J Hematol 2010; 85:921-5.

28. Ustun C, Reiter A, Scott BL, et al. Hematopoietic stem-cell transplantation for advanced systemic mastocytosis. J Clin Oncol 2014; 32:3264-74.

29. Georgin-Lavialle S, Lhermitte L, Dubreuil P, et al. Mast cell leukemia. Blood $2013 ; 121: 1285-95$. 
30. Cherner JA, Jensen RT, Dubois A, O’Dorisio TM, Gardner JD, Metcalfe DD. Gastrointestinal dysfunction in systemic mastocytosis. A prospective study. Gastroenterology 1988; 95:657-667.

31. Lim KH, Tefferi A, Lasho TL, et al; Systemic mastocytosis in 342 consecutive adults: survival studies and prognostic factors. Blood. 2009;113(23):5727-36.

32. Theoharides TC, Valent P, Akin C. Mast cells, mastocytosis, and related disorders. N Engl J Med 2015;373:163-72 


\title{
Relevant Imaging Presentation of Erdheim- Chester Disease
}

By Philippe Jacob, Sana Chambah, and Michèle Dugal MD

\author{
About the Authors \\ Philippe Jacob is a medical student at Laval University. Sana Chambah is an internal medicine resident at Laval University. Michèle \\ Dugal is an internist in the Internal Medicine Department of Hôtel Dieu de Québec Hospital. \\ Corresponding author philippe.jacob.1@ulaval.ca \\ Submitted: November 7, 2017; Accepted: January 2, 2018. Published: June 25, 2018.
}

\begin{abstract}
Erdheim-Chester disease (ECD) is a rare non-Langerhans multisystemic histiocytosis. This disorder is characterized by CD68+/CDa1- foamy histiocytic infiltration of tissues, especially bones, retroperitoneum, heart, lung and brain. Clinical manifestations may range from asymptomatic bone lesions to multiorganic symptoms. Bone pain in lower extremities is the most common symptom. Typical imaging findings include symmetric diaphyseal osteosclerosis of long bones, periaortic sheathing ("coated aorta") and retroperitoneal infiltration ("hairy kidney"). Lung and brain radiological abnormalities may also be seen. BRAF-V600E mutation is present in almost half of this population. Vemurafenib, a mutated BRAF inhibitor, is a promising treatment for patients with this mutation. We present the case of a 60 -year-old man who arrived with a pathological right humerus fracture, initially thought to be tuberculous in nature. ECD was considered after imaging.
\end{abstract}

\section{RÉSUMÉ}

La maladie d'Erdheim-Chester (ECD) est une histiocytose multisystémique rare non langerhansienne. Ce trouble est caractérisé par une infiltration histiocytaire CD68+/CDa1mousseuse des tissus, en particulier des os, du rétropéritoine, du cœur, du poumon et du cerveau. Les manifestations cliniques peuvent aller de lésions osseuses asymptomatiques à des symptômes multiorganiques. La douleur osseuse dans les membres inférieurs est le symptôme le plus commun. Les résultats d'imagerie typiques incluent une ostéosclérose diaphysaire symétrique des os longs, un gainage péri-aortique (" aorte enduite») et une infiltration rétro péritonéale ( rein poilu»). Des anomalies radiologiques du poumon et du cerveau peuvent également être observées. La mutation BRAF-V600E est présente dans près de la moitié de cette population. Le vémurafénib, un inhibiteur de BRAF muté, est un traitement prometteur pour les patients atteints de cette mutation. Nous présentons le cas d'un homme de 60 ans qui est arrivé avec une fracture pathologique de l'humérus droit, initialement considérée comme tuberculeuse. L'ECD a été considérée après l'imagerie.

Erdheim-Chester disease (ECD) is a non-Langerhans histiocytosis, with 600 known cases to date. ${ }^{1}$ First described in 1930 by Jakob Erdheim and William Chester, this disease is characterized by the infiltration of tissues with foamy histiocytes, typically CD68+/
CD1a-, unlike Langerhans cell histiocytosis, which is CD68+/ $\mathrm{CD} 1 \mathrm{a}+.^{2,3}$ The etiology of this condition is unclear. ${ }^{2,4,5}$ This multisystem disorder can lead to a variety of clinical manifestations, such as bone pain, exophthalmos, diabetes insipidus, cerebellar 
syndromes and xanthelasma, particularly in men between 50 and 70 years old. However, patients with ECD may be completely asymptomatic. ${ }^{2,45}$ Symmetric diaphyseal osteosclerosis of long bones especially in lower extremities, periaortic thickening (called "coated aorta" when infiltration involves the thoracoabdominal aorta) and retroperitoneal infiltration with appearance of "hairy kidney" are typical imaging findings of ECD. ${ }^{2,4,6,7}$ Interlobular septal thickening and ground-glass/centrilobular opacities in lung computed tomography (CT) may also be observed, as well as pericardial effusion and myocardial infiltration. Cerebellar or brainstem lesions, dural-based lesions and brain parenchymal lesions also be identified. ${ }^{2,4,6-8}$ Biopsy is needed for diagnosis, and will reveal the typical foamy histiocytic infiltration of tissues, as well as BRAF-V600E mutation in 54\% of ECD patients. ${ }^{1-3,9}$ This mutation is useful for selecting appropriate therapy. Vemurafenib might be more effective than interferon $\alpha$ and other treatment modalities in ECD for BRAF mutations. Further evidence is needed. ${ }^{10,11}$

\section{Case Report}

We report a case of a 60-year-old man who was admitted to the Internal Medicine department for pathological fracture of the right humerus. A CT-scan of the arm raised the suspicion of tubercular disease. The patient presented with a short history of severe pain in the right arm without obvious trauma. Over a period of 3 weeks, he had been aware of a mild discomfort in this arm: he attributed this to the use of crutches since his hip surgery 4 months previously. He had no other symptoms. He was admitted under Family Medicine, and underwent testing. Radiography of the right arm revealed a pathological fracture of the humerus and a heterogenic appearance of the diaphysis was

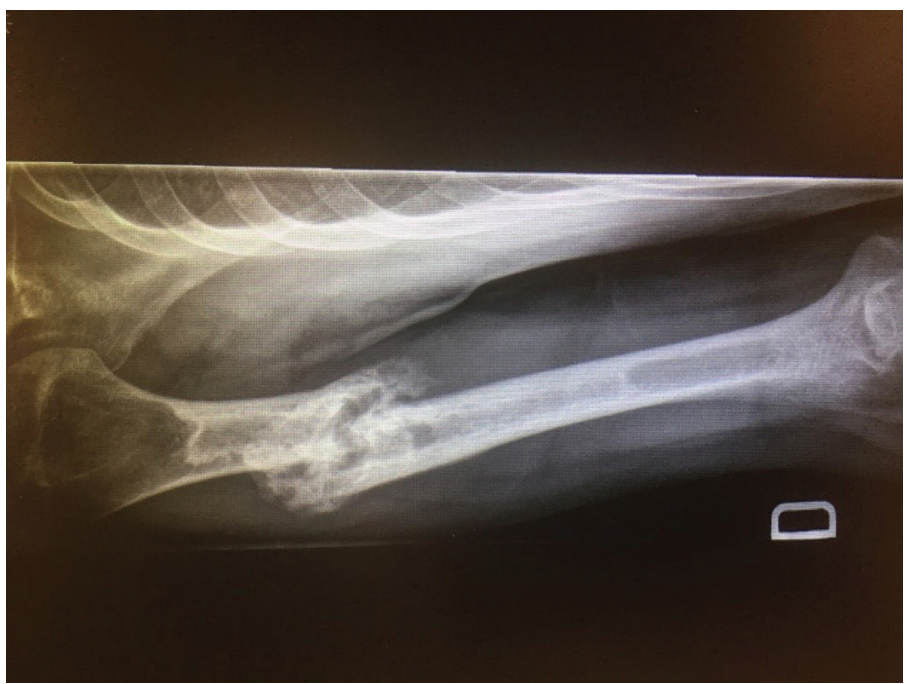

Figure 1. Right humerus pathological fracture. noted (Figure 1). The left hip demonstrated similar diaphyseal anomaly, as well as a $4 \mathrm{~cm}$ lytic lesion (Figure 2). In order to exclude a neoplasia, a CT-scan C+ and a scintigraphy were performed. The preliminary scan report raised the possibility of active tuberculosis: the patient was transferred to Internal Medicine and cared for in a negative pressure room.

This patient's relevant medical history includes left and right hip replacements (in 2014 and 2016 respectively), polyarteritis nodosa at a young age and opioid dependence. He was taking a total of $190 \mathrm{mg}$ of oxycodone per day. He also had $80 \mathrm{mg}$ of testosterone per day on his drug profile for an unknown reason.

The patient had no other symptoms, or findings, of note. Laboratory tests revealed an anemia (hemoglobin 85 to $105 \mathrm{~g} / \mathrm{L}$, MCV 79.8fL, relative reticulocytes 0.011 , iron $6 \mu \mathrm{mol} / \mathrm{L}$, ferritin $204 \mu \mathrm{g} / \mathrm{L}$, transferrin $1.46 \mathrm{~g} / \mathrm{L}$, transferrin saturation 0.16 ), but normal values for leucocytes and platelets. No bone marrow biopsy was performed. His glomerular filtration rate was $60 \mathrm{~mL} /$ min, compared to $86 \mathrm{~mL} / \mathrm{min}$ two years earlier (calculated with Cockcroft-Gault equation). Sodium levels were normal. Inflammatory markers were above the normal range (C-reactive protein $40.73 \mathrm{mg} / \mathrm{L}$, ESR $71 \mathrm{~mm} /$ hour). Ziehl-Neelsen stain was negative in bronchoalveolar lavage (BAL) and Mycobacterium Tuberculosis PCR and culture were negative in blood, urine and BAL.

The CT-scan and the scintigraphy showed multiple abnormalities. In addition to the right humerus fracture seen on X-ray, CT

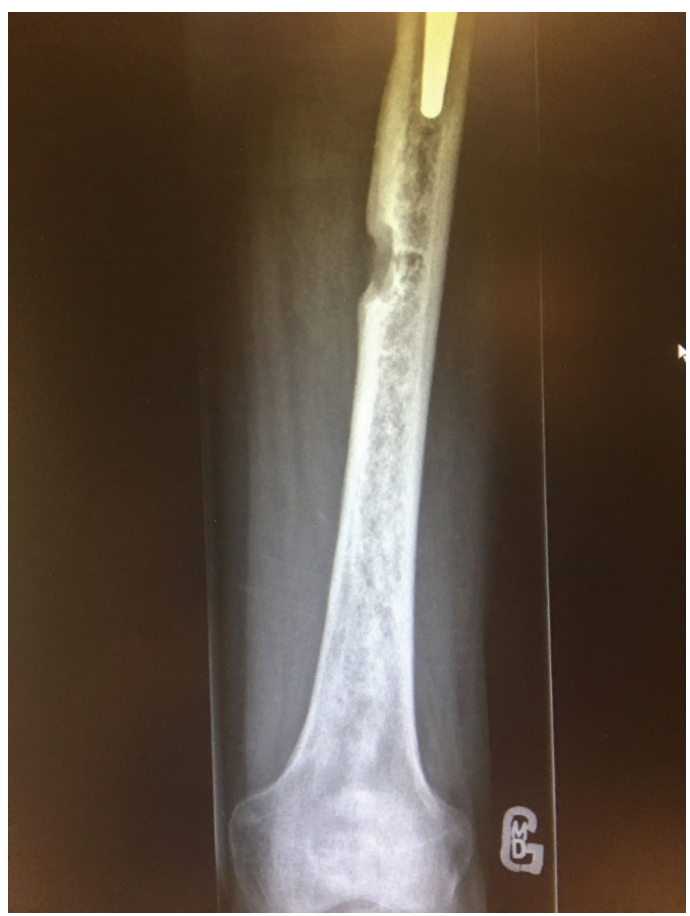

Figure 2. Lytic lesion and heterogenic aspect of the left hip. 
images showed a bone heterogeneity and lytic lesions, as well as a pathological fracture of sacrum. Retroperitoneal infiltration was seen, along with bilateral hydronephrosis (Figure 3). Within the lungs, diffuse and bilateral centrilobular emphysema and pleuroparenchymal apical opacities were seen. Scintigraphy was partially performed, since the patient refused the $2^{\text {nd }}$ part of the test. This early phase study suggested symmetrical accumulation in long bones of both legs and in sacrum alae (Figure 4).

Metastatic X-ray series also showed the heterogenic aspect of both femurs and humeri. Head CT and echocardiography were normal. PET scan showed diffuse sclerosis of the peripheral bone marrow, consistent with ECD.

In order to confirm the diagnosis, a histopathologic review of the right femoral head (removed 4 months earlier when he had hi joint surgery) was requested. Note that pathology report at the time showed severe arthrosis and chronic inflammation with foamy histiocytes attributed to avascular necrosis of femoral head. This review revealed foamy histiocytes CD68+/CD163+/CD1a-, confirming the diagnosis of ECD. Foamy histiocytes were also identified in bronchoalveolar lavage, but immunohistochemistry was not completed. BRAF V600E mutation was positive in the right hip specimen, and Touton giant cells were also seen. Note that PCR for BRAF V600E mutation was negative in blood.

As the BRAF V600E mutation was present, the hematology/ oncology team suggested treatment with vemurafenib $960 \mathrm{mg}$ $\mathrm{q} 12 \mathrm{~h}$ orally. His arm fracture was managed conservatively since the patient refused surgery.

After 6 months follow-up, the patient stayed asymptomatic. He developed a rash after four months of treatment, but it resolved without any intervention. Anemia stayed similar (hemoglobin $97 \mathrm{~g} / \mathrm{L}, \mathrm{MCV}$ 84fL, relative reticulocytes 0.024 , iron $3 \mu \mathrm{mol} / \mathrm{L}$,

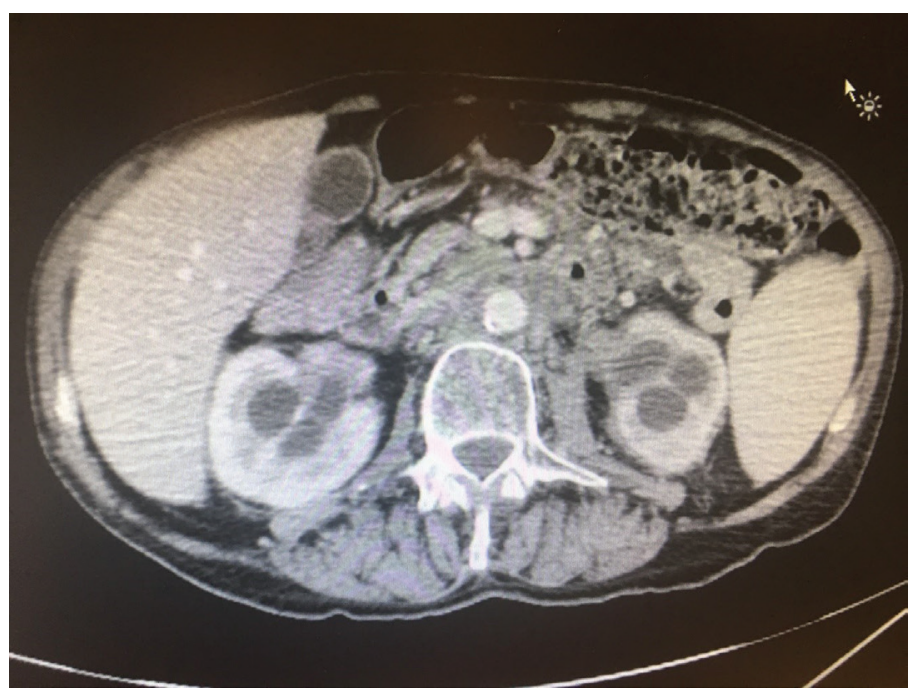

Figure 3. Retroperitoneal infiltration and hydronephrosis at CT-scan. ferritin $239 \mu \mathrm{g} / \mathrm{L}$, transferrin $1.61 \mathrm{~g} / \mathrm{L}$, transferrin saturation 0.07 ) and glomerular filtration dropped to $49 \mathrm{~mL} / \mathrm{min}$ after 3 weeks but then stabilized to his base value $(58 \mathrm{~mL} / \mathrm{min})$. Scintigraphy performed after 2 months of treatment remained unchanged. PET scan was selected as control imaging at 6 months because it has been shown to be the best marker of disease activity and a choice investigation modality during follow-up. ${ }^{2,4,6}$ It revealed a near-normal metabolism of bone structures, a fracture to his right scapula and a right hypermetabolic pleural effusion with hilar ganglions slightly hypermetabolic. These new findings were asymptomatic and the fracture was managed conservatively. Thoracentesis could not be performed since the pleural effusion was too small. A control PET scan is planned after a 12 months follow-up. Note that the patient was not always compliant with treatment even though he did not developed any side effects.

\section{Discussion}

ECD is a rare multisystemic disease which can be a diagnosis challenge. Imaging studies were helpful in this case. According to Célia Autunes et al. ${ }^{4}$, symmetric osteosclerosis of long bones together with perirenal disease or aortic perivascular thickening is almost pathognomonic of ECD. Our patient met some of these criteria: only the aortic infiltration was missing. As a result, the diagnosic probability was high even before tissue analysis. Pulmonary abnormalities found on both CT and bronchoalveolar lavage are non-specific, ${ }^{4,8}$ but consistent with the diagnosis.

It should be noted that bilateral symmetrical osteosclerosis of long bones is present in $74 \%$ of patients ${ }^{1}$, but "coated aorta" and "hairy kidney" are less frequent ( $6 \%$ and $16 \%$ respectively $\left.{ }^{1}\right)$.

Bone pain is common in ECD. ${ }^{1,3-6,8,10}$ Our patient had pain associated with a humeral fracture but did not report any other

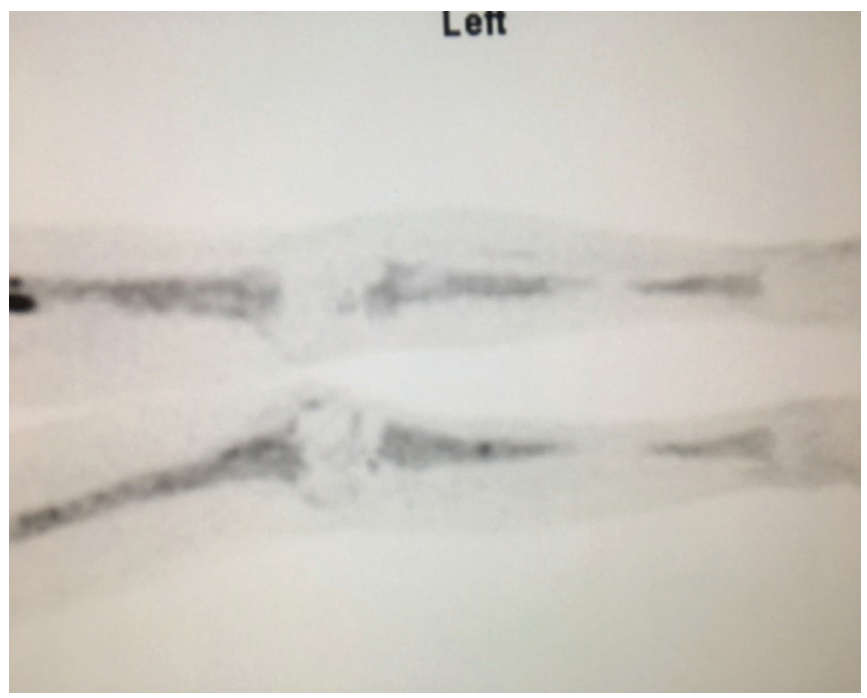

Figure 4. Symmetrical accumulation in long bones of both legs at scintigraphy. 
discomforts despite widespread pathology. This may be due to the high dose of opioid analgesic he was taking. Cough and dyspnea are sometimes associated with $\mathrm{ECD}^{1,5,8}$ when there is lung involvement: our patient didn't have pulmonary involvement. He was also free of neurological symptoms, which are present in 25 to $50 \%$ of cases ${ }^{1}$ in the literature.

Histopathologic review confirmed the diagnosis, and the BRAF-V600E mutation was present in the pathologic specimen. Vemurafenib (a mutated BRAF inhibitor) shows promise in treatment, with more rapid results than interferon $\alpha^{10,11}$ There is a body of evidence in the literature supporting treatment using interferon $a^{1-3,6,12}$ : we chose vemurafenib in the hope of higher efficacy. ${ }^{10,11}$ Severe cutaneous side effects can occur with BRAF inhibitor therapy, such as squamous cell carcinoma and vasculitis; interferon $\alpha$ may be better tolerated. ${ }^{11,13,14}$ Longitudinal follow-up is needed with this treatment. In Quebec, vemurafenib therapy costs $136,56 \$ C A D /$ day and is also used for metastatic melanoma. ${ }^{15}$

After 6 months of treatment, vemurafenib was well tolerated in our patient. Significant improvement was seen on PET scan, since bone metabolism has declined to a near-normal value. However, a pathological fracture to the right scapula and a pleural effusion appeared. Since our patient didn't manifest any symptoms, the age of the fracture is unknown. It might have happened before the clinical effect of vemurafenib occured. Haroche et al. ${ }^{10}$ saw a significant improvement on clinical symptoms, CRP and pathological uptakes on PET scan within four months in their three patients. However, our patient was partially compliant to treatment, so there might have been a delay in efficacy. Pleural effusion could not be characterized since it could not be drained, so the cause remains unclear. PET scan control at 12 months might give more information on vemurafenib efficacy in our patient.

\section{Conclusions}

We present a 60 -year-old man with a pathological fracture with imaging findings suggesting of ECD. There was a paucity of signs and symptoms, other than anemia. Indeed, clinical manifestations are protean and variable. Bone and retroperitoneal abnormalities were key findings for diagnosis in our patient. The mutated BRAF inhibitor vemurafenib may be a promising treatment for patients with BRAF-V600E mutation. However, definitive management guidelines are still evolving.

\section{Conflict of Interests}

None.

\section{Consent Form}

Written consent form was obtained from the patient for publication of this case report.

\section{References}

1. Cives M, Simone V, Rizzo FM, et al. Erdheim-Chester disease: a systematic review. Crit Rev Oncol Hematol 2015 Jul 31;95(1):1-1.

2. Haroche J, Arnaud L, Cohen-Aubart F, et al. Erdheim-Chester disease. Curr Rheumatol Rep 2014 Apr 1;16(4):412.

3. Hervier B, Haroche J, Arnaud L, et al. Association of both Langerhans cell histiocytosis and Erdheim-Chester disease linked to the BRAF V600E mutation. Blood 2014 Aug 14;124(7):1119-26.

4. Antunes C, Graça B, Donato P. Thoracic, abdominal and musculoskeletal involvement in Erdheim-Chester disease: CT, MR and PET imaging findings. Ins Imag 2014 Aug 1;5(4):473-82.

5. Cavalli G, Guglielmi B, Berti A, Campochiaro C, Sabbadini MG, Dagna L. The multifaceted clinical presentations and manifestations of ErdheimChester disease: comprehensive review of the literature and of 10 new cases. Ann Rheumat Diseas 2013 Feb 1:annrheumdis-2012.

6. Diamond EL, Dagna L, Hyman DM, et al. Consensus guidelines for the diagnosis and clinical management of Erdheim-Chester disease. Blood 2014 Jul 24;124(4):483-92.

7. Brun AL, Touitou-Gottenberg D, Haroche J, et al. Erdheim-Chester disease: CT findings of thoracic involvement. Eur Radiol 2010 Nov 1;20(11):2579-87.

8. Arnaud L, Pierre I, Beigelman-Aubry C, et al. Pulmonary involvement in Erdheim-Chester disease: A single-center study of thirty-four patients and a review of the literature. Arthr Rheumatol 2010 Nov 1;62(11):3504-12.

9. Haroche J, Charlotte F, Arnaud L, et al. High prevalence of BRAF V600E mutations in Erdheim-Chester disease but not in other non-Langerhans cell histiocytoses. Blood 2012 Sep 27;120(13):2700-3.

10. Haroche J, Cohen-Aubart F, Emile JF, et al. Dramatic efficacy of vemurafenib in both multisystemic and refractory Erdheim-Chester disease and Langerhans cell histiocytosis harboring the BRAF V600E mutation. Blood 2013 Feb 28;121(9):1495-500.

11. Haroche J, Cohen-Aubart F, Emile JF, et al. Reproducible and sustained efficacy of targeted therapy with vemurafenib in patients with BRAFV600Emutated Erdheim-Chester disease. J Clin Oncol 2014 Nov 24;33(5):411-8.

12. Haroche J, Amoura Z, Trad SG, et al. Variability in the efficacy of interferon- $\alpha$ in Erdheim-Chester disease by patient and site of involvement: Results in eight patients. Arth Rheumatol 2006 Oct 1;54(10):3330-6.

13. Boussemart L, Routier E, Mateus C, et al. Prospective study of cutaneous side-effects associated with the BRAF inhibitor vemurafenib: a study of 42 patients. Ann Oncol 2013 Feb 13;24(6):1691-7.

14. Mirouse A, Savey L, Domont F, et al. Systemic vasculitis associated with vemurafenib treatment: Case report and literature review. Medicine 2016 Nov;95(46).

15. Gouvernement du Québec. Liste des médicaments. Bibliothèque et Archives nationales du Québec. 2017 April 1: 486. 